\title{
Numerical Solution of Eikonal Equation Using Finite Difference Method
}

\section{Sana A. Alotaibi ${ }^{1}$, Arshad M.Younas ${ }^{2}$}

1,2Department Mathematics, King Abdulaziz University, Rabigh, Kingdom of Saudi Arabia

\begin{abstract}
In this paper, a method to calculate tsunami wave front is introduced using the finite difference method to solve the ill-posed problem and to calculate perturbed velocity of the wave front. Comparison between the actual and approximate solution will be proposed in a table form and a graphic form.
\end{abstract}

\section{Keywords}

Wave Propagation, Eikonal Equation, Wave Front, Partial Differential Equation, Analytical Solutions, Tsunami Wave.

\section{Introduction}

Recently, many applications of eikonal equation have been introduced. In1987, Hitashi Ishii published a paper in the proceeding of the American mathematical Society regarding the Hamilton-Jacobi equations of eikonal type: $H(x, u, D u)=0$, in $\Omega$ where $\Omega$ is an open subset of $R^{N}$,

$$
H: \Omega \times R \times R^{N} \rightarrow R, u \in C(\Omega) \text { and denote the gradient of } u \text { by }|D u| .[\mathbf{1}]
$$

Also, E.D. Moskalensky who used two-dimensional eikonal equation. Describing the front of a disturbance propagating. [2],[3]

$$
\left(\frac{\partial f}{\partial x}\right)^{2}+\left(\frac{\partial f}{\partial y}\right)^{2}=\frac{1}{v^{2}(x, y)}
$$

Analytical solutions for the tsunami wave-front behavior above an uneven bottom can be described by the 2-D eikonal equation

$$
\left(\frac{\partial f}{\partial x}\right)^{2}+\left(\frac{\partial f}{\partial y}\right)^{2}=\frac{1}{v^{2}(x, y)}
$$

where $v(x, y)$ is the wave propagation velocity distribution in the environment. The position of a wave front at the time is given by the equation

$$
f(x, y)=c
$$

As a matter of fact, solutions of equation (1) are known only for rather a limited number of functions $v$, therefore usually the wave front position is obtained by numerical methods.[4] we will use the finite difference method to obtain the position of wave front.

\section{Finite Difference Method}

The finite Difference Method for solving differential equations is simple to understand and implement. However, it has one significant drawback: it can only be applied to meshes in which the cell faces are lined up with the coordinate axes. As such it becomes difficult, if not outright impossible to resolve curved boundaries- like those encountered when dealing with any realistic geometry. [5],[6] 


\section{The two- dimensional Eikonal equation is:}

$$
f_{x}^{2}+f_{y}^{2}=\emptyset^{2}
$$

Where:

$\varnothing=\frac{1}{v}$ and $v(x, y)$ the wave propagation velocity

$$
\left(\frac{\partial \mathrm{f}}{\partial \mathrm{x}}\right)^{2}+\left(\frac{\partial f}{\partial y}\right)^{2}=\frac{1}{v^{2}(\mathrm{x}, \mathrm{y})}
$$

The Eikonal equation using finite difference method:

$$
\begin{aligned}
& \left(\frac{f_{i+1, j}-f_{i, j}}{h}\right)^{2}+\left(\frac{f_{i, j+1}-f_{i, j}}{k}\right)^{2}=\emptyset^{2}\left(x_{i}, y_{j}\right) \\
& \left(\frac{f_{i, j+1}-f_{i, j}}{k}\right)^{2}=\emptyset^{2}\left(x_{i}, y_{j}\right)-\left(\frac{f_{i+1, j}-f_{i, j}}{h}\right)^{2} \\
& \frac{f_{i, j+1}^{2}-2 * f_{i, j} * f_{i, j+1}+f_{i, j}^{2}}{k^{2}}=\emptyset^{2}\left(x_{i}, y_{j}\right)-\frac{\left(f_{i+1, j}^{2}-2 * f_{i, j} * f_{i+1, j}+f_{i, j}^{2}\right)}{h^{2}} \\
& f_{i . j+1}=\sqrt{k^{2} * \emptyset^{2}\left(x_{i}, y_{j}\right)+2 * f_{i, j} * f_{i, j+1}-f_{i, j}^{2}+\frac{k^{2}}{h^{2}} *\left(-f_{i+1, j}^{2}+2 * f_{i, j} * f_{i+1, j}-f_{i, j}^{2}\right)} \\
& f_{i . j+1}=\sqrt{k^{2} * \emptyset^{2}\left(x_{i}, y_{j}\right)+2 * f_{i, j} * f_{i, j+1}-\frac{k^{2}}{h^{2}} * f_{i+1, j}^{2}+2 * \frac{k^{2}}{h^{2}} * f_{i, j} * f_{i+1, j}-\left(1+\frac{k^{2}}{h^{2}}\right) * f_{i, j}^{2}}
\end{aligned}
$$

when $\emptyset(x, y)=\frac{\sqrt{x^{2}+y^{2}}}{\sqrt{(x+1)^{2}+y^{2}}}$,

exact solution $=x-\frac{1}{2} * \ln \left((x+1)^{2}+y^{2}\right)$

when $\varnothing(x, y)=\frac{1}{|x|}$

exact solution $=-\log \left(\frac{\sqrt{x^{2}+y^{2}}+y}{x}\right)$, for $\boldsymbol{x}>\mathbf{0}$

\section{Numerical Results of Finite Difference Method}

The numerical results of the finite difference method illustrated in the following tables corresponding to different selected values of $i, j$ and $h$ Where $i=1,2,3,4 ; j=0,1,2, \ldots, 30$; with a corresponding step size $(h=0.2)$ and where $i=1,2,3, \ldots, 9 ; j=0,1,2, \ldots, 1$; with a corresponding step size $(h=0.1)$ illustrated in Table.(1) and Table.(2), respectively.

3.1 The Results when $\varnothing(x, y)=\frac{\sqrt{x^{2}+y^{2}}}{\sqrt{(x+1)^{2}+y^{2}}}$ [2] 
Table. (1): Numerical results of the 2-D Eikonal equation using F.D.M when $\emptyset(x, y)=\frac{\sqrt{x^{2}+y^{2}}}{\sqrt{(x+1)^{2}+y^{2}}}$

\begin{tabular}{|c|c|c|c|c|c|c|}
\hline $\bar{i} \boldsymbol{i}$ & $\bar{j} \boldsymbol{j}$ & $\bar{X} \boldsymbol{X}(\boldsymbol{i})$ & $\bar{Y}(j)$ & $\bar{W}$ & $\bar{U}$ & $\bar{E}$ \\
\hline \multirow[t]{31}{*}{1} & 0 & 1.2 & 1 & 0.3176346015 & 0.3176346015 & 0.0000000000 \\
\hline & 1 & 1.2 & 1.03 & 0.3019791618 & 0.3118651250 & 0.0098859632 \\
\hline & 2 & 1.2 & 1.06 & 0.2869939907 & 0.3059755550 & 0.0189815643 \\
\hline & 3 & 1.2 & 1.10 & 0.2726192591 & 0.2999708640 & 0.0273516049 \\
\hline & 4 & 1.2 & 1.13 & 0.2588118878 & 0.2938559755 & 0.0350440877 \\
\hline & 5 & 1.2 & 1.16 & 0.2455422899 & 0.2876357560 & 0.0420934661 \\
\hline & 6 & 1.2 & 1.20 & 0.2327917355 & 0.2813150100 & 0.0485232745 \\
\hline & 7 & 1.2 & 1.23 & 0.2205502103 & 0.2748984675 & 0.0543482572 \\
\hline & 8 & 1.2 & 1.26 & 0.2088146611 & 0.2683907835 & 0.0595761224 \\
\hline & 9 & 1.2 & 1.30 & 0.1975875570 & 0.2617965285 & 0.0642089715 \\
\hline & 10 & 1.2 & 1.33 & 0.1868757077 & 0.2551201850 & 0.0682444773 \\
\hline & 11 & 1.2 & 1.36 & 0.1766892972 & 0.2483661420 & 0.0716768448 \\
\hline & 12 & 1.2 & 1.40 & 0.1670411039 & 0.2415386940 & 0.0744975901 \\
\hline & 13 & 1.2 & 1.43 & 0.1579458869 & 0.2346420330 & 0.0766961461 \\
\hline & 14 & 1.2 & 1.46 & 0.1494199205 & 0.2276802495 & 0.0782603290 \\
\hline & 15 & 1.2 & 1.50 & 0.1414806722 & 0.2206573295 & 0.0791766573 \\
\hline & 16 & 1.2 & 1.53 & 0.1341466160 & 0.2135771525 & 0.0794305365 \\
\hline & 17 & 1.2 & 1.56 & 0.1274371827 & 0.2064434885 & 0.0790063058 \\
\hline & 18 & 1.2 & 1.60 & 0.1213728482 & 0.1992600000 & 0.0778871518 \\
\hline & 19 & 1.2 & 1.63 & 0.1159753668 & 0.1920302400 & 0.0760548732 \\
\hline & 20 & 1.2 & 1.66 & 0.1112681614 & 0.1847576520 & 0.0734894906 \\
\hline & 21 & 1.2 & 1.70 & 0.1072768845 & 0.1774455680 & 0.0701686835 \\
\hline & 22 & 1.2 & 1.73 & 0.1040301734 & 0.1700972160 & 0.0660670426 \\
\hline & 23 & 1.2 & 1.76 & 0.1015606301 & 0.1627157120 & 0.0611550819 \\
\hline & 24 & 1.2 & 1.80 & 0.0999060656 & 0.1553040640 & 0.0553979984 \\
\hline & 25 & 1.2 & 1.83 & 0.0991110647 & 0.1478651770 & 0.0487541123 \\
\hline & 26 & 1.2 & 1.86 & 0.0992289422 & 0.1404018500 & 0.0411729078 \\
\hline & 27 & 1.2 & 1.90 & 0.1003241881 & 0.1329167800 & 0.0325925919 \\
\hline & 28 & 1.2 & 1.93 & 0.1024755256 & 0.1254125600 & 0.0229370344 \\
\hline & 29 & 1.2 & 1.96 & 0.1057797498 & 0.1178916880 & 0.0121119382 \\
\hline & 30 & 1.2 & 2 & 0.1103565620 & 0.1103565620 & 0.0000000000 \\
\hline
\end{tabular}




\begin{tabular}{|c|c|c|c|c|c|c|}
\hline \multirow[t]{31}{*}{2} & 0 & 1.4 & 1 & 0.4444885550 & 0.4444885550 & 0.0000000000 \\
\hline & 1 & 1.4 & 1.03 & 0.4253273535 & 0.4395003705 & 0.0141730170 \\
\hline & 2 & 1.4 & 1.06 & 0.4069293542 & 0.4344003505 & 0.0274709963 \\
\hline & 3 & 1.4 & 1.10 & 0.3892903849 & 0.4291923875 & 0.0399020026 \\
\hline & 4 & 1.4 & 1.13 & 0.3724102382 & 0.4238803580 & 0.0514701198 \\
\hline & 5 & 1.4 & 1.16 & 0.3562929077 & 0.4184681160 & 0.0621752083 \\
\hline & 6 & 1.4 & 1.20 & 0.3409465299 & 0.4129594870 & 0.0720129571 \\
\hline & 7 & 1.4 & 1.23 & 0.3263831215 & 0.4073582620 & 0.0809751405 \\
\hline & 8 & 1.4 & 1.26 & 0.3126181761 & 0.4016681925 & 0.0890500164 \\
\hline & 9 & 1.4 & 1.30 & 0.2996701807 & 0.3958929840 & 0.0962228033 \\
\hline & 10 & 1.4 & 1.33 & 0.2875600912 & 0.3900362930 & 0.1024762018 \\
\hline & 11 & 1.4 & 1.36 & 0.2763108058 & 0.3841017220 & 0.1077909162 \\
\hline & 12 & 1.4 & 1.40 & 0.2659466616 & 0.3780928180 & 0.1121461564 \\
\hline & 13 & 1.4 & 1.43 & 0.2564929791 & 0.3720130640 & 0.1155200849 \\
\hline & 14 & 1.4 & 1.46 & 0.2479756699 & 0.3658658800 & 0.1178902101 \\
\hline & 15 & 1.4 & 1.50 & 0.2404209214 & 0.3596546200 & 0.1192336986 \\
\hline & 16 & 1.4 & 1.53 & 0.2338549672 & 0.3533825680 & 0.1195276008 \\
\hline & 17 & 1.4 & 1.56 & 0.2283039486 & 0.3470529390 & 0.1187489904 \\
\hline & 18 & 1.4 & 1.60 & 0.2237938693 & 0.3406688720 & 0.1168750027 \\
\hline & 19 & 1.4 & 1.63 & 0.2203506407 & 0.3342334360 & 0.1138827953 \\
\hline & 20 & 1.4 & 1.66 & 0.2180002132 & 0.3277496200 & 0.1097494068 \\
\hline & 21 & 1.4 & 1.70 & 0.2167687842 & 0.3212203400 & 0.1044515558 \\
\hline & 22 & 1.4 & 1.73 & 0.2166830682 & 0.3146484340 & 0.0979653658 \\
\hline & 23 & 1.4 & 1.76 & 0.2177706108 & 0.3080366630 & 0.0902660522 \\
\hline & 24 & 1.4 & 1.80 & 0.2200601215 & 0.3013877120 & 0.0813275905 \\
\hline & 25 & 1.4 & 1.83 & 0.2235817927 & 0.2947041860 & 0.0711223933 \\
\hline & 26 & 1.4 & 1.86 & 0.2283675609 & 0.2879886140 & 0.0596210531 \\
\hline & 27 & 1.4 & 1.90 & 0.2344512564 & 0.2812434520 & 0.0467921956 \\
\hline & 28 & 1.4 & 1.93 & 0.2418685678 & 0.2744710740 & 0.0326025062 \\
\hline & 29 & 1.4 & 1.96 & 0.2506567285 & 0.2676737800 & 0.0170170515 \\
\hline & 30 & 1.4 & 2 & 0.2608538000 & 0.2608538000 & 0.0000000000 \\
\hline \multirow[t]{2}{*}{3} & 0 & 1.6 & 1 & 0.5755088330 & 0.5755088330 & 0.0000000000 \\
\hline & 1 & 1.6 & 1.03 & 0.5567670515 & 0.5711606700 & 0.0143936185 \\
\hline
\end{tabular}




\begin{tabular}{|c|c|c|c|c|c|c|}
\hline & 2 & 1.6 & 1.06 & 0.5388356746 & 0.5667092870 & 0.0278736124 \\
\hline & 3 & 1.6 & 1.10 & 0.5217044149 & 0.5621577540 & 0.0404533391 \\
\hline & 4 & 1.6 & 1.13 & 0.5053674542 & 0.5575091390 & 0.0521416848 \\
\hline & 5 & 1.6 & 1.16 & 0.4898237435 & 0.5527665100 & 0.0629427665 \\
\hline & 6 & 1.6 & 1.20 & 0.4750769592 & 0.5479329230 & 0.0728559638 \\
\hline & 7 & 1.6 & 1.23 & 0.4611352224 & 0.5430114240 & 0.0818762016 \\
\hline & 8 & 1.6 & 1.26 & 0.4480106623 & 0.5380050410 & 0.0899943787 \\
\hline & 9 & 1.6 & 1.30 & 0.4357188857 & 0.5329167800 & 0.0971978943 \\
\hline & 10 & 1.6 & 1.33 & 0.4242784065 & 0.5277496200 & 0.1034712135 \\
\hline & 11 & 1.6 & 1.36 & 0.4137100666 & 0.5225065140 & 0.1087964474 \\
\hline & 12 & 1.6 & 1.40 & 0.4040364855 & 0.5171903810 & 0.1131538955 \\
\hline & 13 & 1.6 & 1.43 & 0.3952815585 & 0.5118041050 & 0.1165225465 \\
\hline & 14 & 1.6 & 1.46 & 0.3874700195 & 0.5063505310 & 0.1188805115 \\
\hline & 15 & 1.6 & 1.50 & 0.3806270864 & 0.5008324640 & 0.1202053776 \\
\hline & 16 & 1.6 & 1.53 & 0.3747781945 & 0.4952526650 & 0.1204744705 \\
\hline & 17 & 1.6 & 1.56 & 0.3699488227 & 0.4896138500 & 0.1196650273 \\
\hline & 18 & 1.6 & 1.60 & 0.3661644177 & 0.4839186860 & 0.1177542683 \\
\hline & 19 & 1.6 & 1.63 & 0.3634504105 & 0.4781697930 & 0.1147193825 \\
\hline & 20 & 1.6 & 1.66 & 0.3618323211 & 0.4723697400 & 0.1105374189 \\
\hline & 21 & 1.6 & 1.70 & 0.3613359428 & 0.4665210420 & 0.1051850992 \\
\hline & 22 & 1.6 & 1.73 & 0.3619875911 & 0.4606261650 & 0.0986385739 \\
\hline & 23 & 1.6 & 1.76 & 0.3638143995 & 0.4546875170 & 0.0908731175 \\
\hline & 24 & 1.6 & 1.80 & 0.3668446358 & 0.4487074540 & 0.0818628182 \\
\hline & 25 & 1.6 & 1.83 & 0.3711080074 & 0.4426882740 & 0.0715802666 \\
\hline & 26 & 1.6 & 1.86 & 0.3766359127 & 0.4366322230 & 0.0599963103 \\
\hline & 27 & 1.6 & 1.90 & 0.3834615843 & 0.4305414890 & 0.0470799047 \\
\hline & 28 & 1.6 & 1.93 & 0.3916200518 & 0.4244182020 & 0.0327981502 \\
\hline & 29 & 1.6 & 1.96 & 0.4011478315 & 0.4182644400 & 0.0171166085 \\
\hline & 30 & 1.6 & 2 & 0.4120822220 & 0.4120822220 & 0.0000000000 \\
\hline 4 & 0 & 1.8 & 1 & 0.7103565620 & 0.7103565620 & 0.0000000000 \\
\hline & 1 & 1.8 & 1.03 & 0.6961652382 & 0.7065375980 & 0.0103723598 \\
\hline & 2 & 1.8 & 1.06 & 0.6827772816 & 0.7026238060 & 0.0198465244 \\
\hline & 3 & 1.8 & 1.10 & 0.6701093050 & 0.6986176210 & 0.0285083160 \\
\hline
\end{tabular}




\begin{tabular}{||c||c|c|c|c|c|}
\hline 4 & 1.8 & 1.13 & 0.6580988462 & 0.6945214920 & 0.0364226458 \\
\hline 5 & 1.8 & 1.16 & 0.6467003138 & 0.6903378750 & 0.0436375612 \\
\hline 6 & 1.8 & 1.20 & 0.6358817230 & 0.6860692260 & 0.0501875030 \\
\hline 7 & 1.8 & 1.23 & 0.6256220504 & 0.6817180040 & 0.0560959536 \\
\hline 8 & 1.8 & 1.26 & 0.6159090772 & 0.6772866600 & 0.0613775828 \\
\hline 9 & 1.8 & 1.30 & 0.6067376257 & 0.6727776410 & 0.0660400153 \\
\hline 10 & 1.8 & 1.33 & 0.5981081154 & 0.6681933810 & 0.0700852656 \\
\hline 11 & 1.8 & 1.36 & 0.5900253820 & 0.6635363020 & 0.0735109200 \\
\hline 12 & 1.8 & 1.40 & 0.5824977220 & 0.6588088070 & 0.0763110850 \\
\hline 13 & 1.8 & 1.43 & 0.5755361314 & 0.6540132840 & 0.0784771526 \\
\hline 14 & 1.8 & 1.46 & 0.5691537192 & 0.6491520960 & 0.0799983768 \\
\hline 15 & 1.8 & 1.50 & 0.5633652809 & 0.6442275830 & 0.0808623021 \\
\hline 16 & 1.8 & 1.53 & 0.5581870226 & 0.6392420600 & 0.0810550374 \\
\hline 17 & 1.8 & 1.56 & 0.5536364335 & 0.6341978120 & 0.0805613785 \\
\hline 18 & 1.8 & 1.60 & 0.5497323077 & 0.6290970970 & 0.0793647893 \\
\hline 19 & 1.8 & 1.63 & 0.5464949157 & 0.6239421380 & 0.0774472223 \\
\hline 20 & 1.8 & 1.66 & 0.5439463405 & 0.6187351280 & 0.0747887875 \\
\hline 21 & 1.8 & 1.70 & 0.5421109886 & 0.6134782220 & 0.0713672334 \\
\hline 22 & 1.8 & 1.73 & 0.5410163008 & 0.6081735420 & 0.0671572412 \\
\hline 23 & 1.8 & 1.76 & 0.5406936874 & 0.6028231720 & 0.0621294846 \\
\hline 24 & 1.8 & 1.80 & 0.5411797335 & 0.5974291600 & 0.0562494265 \\
\hline 25 & 1.8 & 1.83 & 0.5425177236 & 0.5919935100 & 0.0494757864 \\
\hline 26 & 1.8 & 1.86 & 0.5447595595 & 0.5865181920 & 0.0417586325 \\
\hline 27 & 1.8 & 1.90 & 0.5479681650 & 0.5810051350 & 0.0330369700 \\
\hline 28 & 1.8 & 1.93 & 0.5522205045 & 0.5754562240 & 0.0232357195 \\
\hline 29 & 1.8 & 1.96 & 0.5576113741 & 0.5698733060 & 0.0122619319 \\
\hline 30 & 1.8 & 2 & 0.5642581860 & 0.5642581860 & 0.00000000000 \\
\hline
\end{tabular}

3.2 The Results when $\emptyset=1 /|x|$ 
Table. (2): Numerical results of the 2-D Eikonal equation using F.D.M when $\emptyset=\mathbf{1} /|\boldsymbol{x}|$

\begin{tabular}{|c|c|c|c|c|c|c|}
\hline$i$ & $\boldsymbol{j}$ & $\boldsymbol{X}(\boldsymbol{i})$ & $Y(j)$ & $W$ & $\boldsymbol{U}$ & $\boldsymbol{E}$ \\
\hline \multirow[t]{11}{*}{1} & 0 & 1.1 & 1 & -0.815608901 & -0.81560890 & 0.000000000 \\
\hline & 1 & 1.1 & 1.1 & -0.903569533 & -0.88137359 & 0.022195946 \\
\hline & 2 & 1.1 & 1.2 & -0.978699255 & -0.94421786 & 0.034481392 \\
\hline & 3 & 1.1 & 1.3 & -1.045743821 & -1.00428118 & 0.041462646 \\
\hline & 4 & 1.1 & 1.4 & -1.106819220 & -1.06171232 & 0.045106898 \\
\hline & 5 & 1.1 & 1.5 & -1.162945528 & -1.11666280 & 0.046282733 \\
\hline & 6 & 1.1 & 1.6 & -1.214532568 & -1.16928220 & 0.045250371 \\
\hline & 7 & 1.1 & 1.7 & -1.261481324 & -1.21971524 & 0.041766083 \\
\hline & 8 & 1.1 & 1.8 & -1.303036613 & -1.26809990 & 0.034936713 \\
\hline & 9 & 1.1 & 1.9 & -1.337235593 & -1.31456638 & 0.022669211 \\
\hline & 10 & 1.1 & 2 & -1.359236682 & -1.35923668 & 0.000000000 \\
\hline \multirow[t]{11}{*}{2} & 0 & 1.2 & 1 & -0.758486137 & -0.75848614 & 0.000000000 \\
\hline & 1 & 1.2 & 1.1 & -0.853257751 & -0.82120395 & 0.032053802 \\
\hline & 2 & 1.2 & 1.2 & -0.933399301 & -0.88137359 & 0.052025714 \\
\hline & 3 & 1.2 & 1.3 & -1.003131280 & -0.93908906 & 0.064042216 \\
\hline & 4 & 1.2 & 1.4 & -1.064923635 & -0.99445749 & 0.070466141 \\
\hline & 5 & 1.2 & 1.5 & -1.120108734 & -1.04759301 & 0.072515721 \\
\hline & 6 & 1.2 & 1.6 & -1.169195745 & -1.09861229 & 0.070583456 \\
\hline & 7 & 1.2 & 1.7 & -1.211942911 & -1.14763132 & 0.064311594 \\
\hline & 8 & 1.2 & 1.8 & -1.247221574 & -1.19476322 & 0.052458357 \\
\hline & 9 & 1.2 & 1.9 & -1.272630303 & -1.24011680 & 0.032513508 \\
\hline & 10 & 1.2 & 2 & -1.283795663 & -1.28379566 & 0.000000000 \\
\hline 3 & 0 & 1.3 & 1 & -0.708460844 & -0.70846084 & 0.000000000 \\
\hline
\end{tabular}




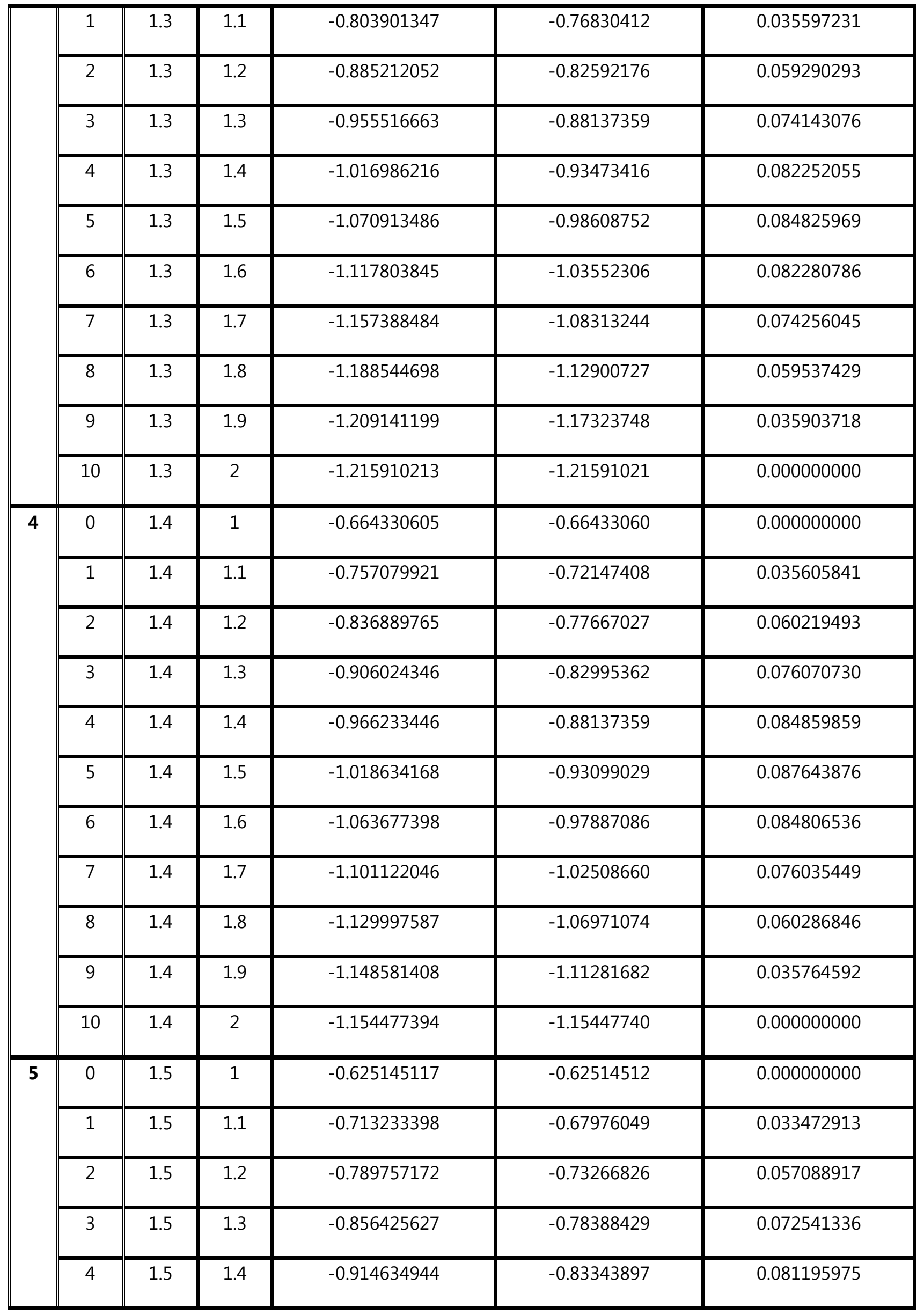









\begin{tabular}{|c|c|c|c|c|c|c|}
\hline & 9 & 1.7 & 1.9 & -0.987364843 & -0.96216567 & 0.025199171 \\
\hline & 10 & 1.7 & 2 & -1.000822379 & -1.00082238 & 0.000000000 \\
\hline \multirow[t]{11}{*}{8} & 0 & 1.8 & $\overline{1}$ & -0.530342598 & -0.53034260 & 0.000000000 \\
\hline & 1 & 1.8 & 1.1 & -0.597294307 & -0.57832922 & 0.018965090 \\
\hline & 2 & 1.8 & 1.2 & -0.657172861 & -0.62514512 & 0.032027744 \\
\hline & 3 & 1.8 & 1.3 & -0.711291526 & -0.67077669 & 0.040514840 \\
\hline & 4 & 1.8 & 1.4 & -0.760504334 & -0.71522142 & 0.045282911 \\
\hline & 5 & 1.8 & 1.5 & -0.805309808 & -0.75848614 & 0.046823671 \\
\hline & 6 & 1.8 & 1.6 & -0.845894790 & -0.80058527 & 0.045309524 \\
\hline & 7 & 1.8 & 1.7 & -0.882134484 & -0.84153933 & 0.040595154 \\
\hline & 8 & 1.8 & 1.8 & -0.913557556 & -0.88137359 & 0.032183969 \\
\hline & 9 & 1.8 & 1.9 & -0.939267014 & -0.92011684 & 0.019150174 \\
\hline & 10 & 1.8 & 2 & -0.957800449 & -0.95780045 & 0.000000000 \\
\hline \multirow[t]{11}{*}{9} & 0 & 1.9 & 1 & -0.504624665 & -0.50462467 & 0.000000000 \\
\hline & 1 & 1.9 & 1.1 & -0.561711042 & -0.55068879 & 0.011022247 \\
\hline & 2 & 1.9 & 1.2 & -0.613904580 & -0.59571437 & 0.018190210 \\
\hline & 3 & 1.9 & 1.3 & -0.662429908 & -0.63968336 & 0.022746550 \\
\hline & 4 & 1.9 & 1.4 & -0.707880160 & -0.68258761 & 0.025292551 \\
\hline & 5 & 1.9 & 1.5 & -0.750554946 & -0.72442747 & 0.026127480 \\
\hline & 6 & 1.9 & 1.6 & -0.790559118 & -0.76521040 & 0.025348721 \\
\hline & 7 & 1.9 & 1.7 & -0.827817822 & -0.80494974 & 0.022868083 \\
\hline & 8 & 1.9 & 1.8 & -0.862039585 & -0.84366355 & 0.018376036 \\
\hline & 9 & 1.9 & 1.9 & -0.892590064 & -0.88137359 & 0.011216476 \\
\hline & 10 & 1.9 & 2 & -0.918104422 & -0.91810442 & 0.000000000 \\
\hline
\end{tabular}




\section{Graph of Eikonal Equation Using F.D.M}

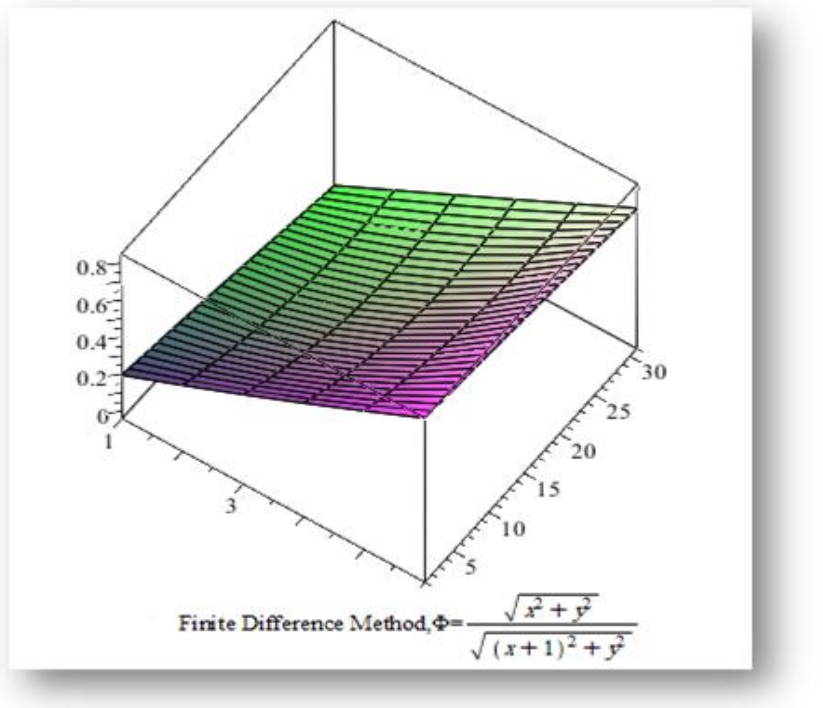

Figure 1. Plot of the 2-D Eikonal equation using F.D.M when $\emptyset(x, y)=\frac{\sqrt{x^{2}+y^{2}}}{\sqrt{(x+1)^{2}+y^{2}}}$

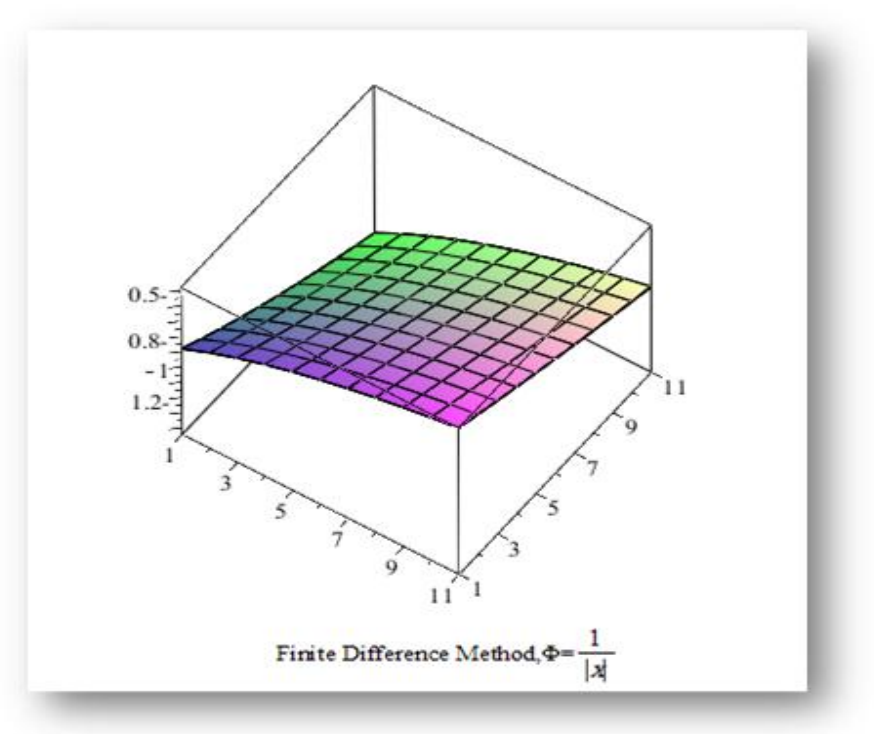

Figure 2. Plot of the 2-D Eikonal equation using F.D.M when $\emptyset=1 /|x|$

\section{Graphs of the Comparison Between Actual Solution and Approximate Solution}

\section{Conclusion}

The purpose of this paper is to represent the solution of two-dimensional Eikonal equation using finite difference method. The results obtained by this method can by described as good results and that was achieved by applying the comparison between the results of the actual solution and the approximate solution.

Also, by adding more information about a particular sea, we can save human beings from the distractive effects due to tsunami. Finally, earthquakes can be fore cared using this dynamical case.

\section{ACKNOWLEDGEMENT}

The author wishes to gratefully acknowledge the referees of this paper who helped to improve the presentation.

\section{REFERENCES}

1. Hitoshi Ishii, A Simple, Direct Proof of Uniqueness for So-lutions of the Hamilton-Jacobi Equations of Eikonal Type,1987.

2. E.D.Moskalensky, Finding exact solution to the Two-Dimensional eikonal equation, numerical analysis and applications,2009.

3. Marchuk, An.G., Chubarov, L.B., and Shokin, Yu.I., Chislennoe modelirovanie voln tsunami (Numerical Simulation of Tsunami Waves), Novosibirsk: Nauka, 1983.

4. An.G. Marchuk, E.D. Moskalensky, Some analytical solutions for tsunami wave rays and front,2010.

5. J.W.Thomas,Numerical Partial differential equation, spring science + business,inc,new York,1995. 